\title{
Reduce energy loss with dynamic positioning controller for USV based on Hierarchical Sliding Mode Control
}

\author{
T.K. Nguyen ${ }^{1}, T h . T r . \mathrm{Le}^{2}{ }^{*}$ and C.X. Nguyen ${ }^{3}$ \\ ${ }^{1}$ Electrical Engineering Department, Vinh University of Technology Education, 117 Nguyen Viet \\ Xuan Street, Vinh city, Vietnam \\ ${ }^{2}$ Controls, Automation in Production and Improvement of Technology Institute, 89B Ly Nam De \\ Street, Hanoi city, Vietnam \\ ${ }^{3}$ Department of Automation and Computing Techniques, Le Quy Don Technical University, Vietnam
}

\begin{abstract}
This paper presents a positional stabilization controller for the under-actuators USV model based on a Hierarchical Sliding Mode Control System (HSMC) that takes into account environmental influences when operating as the flow, waves and wind. The controller presents successful simulation results demonstrating stability in the presence of noise. It helps to reduce energy loss in human activities at the sea.
\end{abstract}

\section{Introduction}

Water surface occupies a very large area of the Earth. Therefore, the demand of transport, construction, rescue and rescue, exploration, and military service ... is great, so improving the quality of control for marine vehicles will greatly reduce energy loss in human activities at sea. In recent years, Unmanned Surface Vehicle (USV) attracts a lot of attention from scientists. Controlling the USV is a major challenge for researchers because of the strong and complex nonlinearity of the itself model and the operating environment. There have been some studies on linear [11] and nonlinear control of USV, boats and in articles [1], [3], [4], [5].

Sliding mode control is a nonlinear controller with remarkable properties is rapid response, less sensitive to parameter changes and turbulence, so it is widely used to design controllers for systems with uncertainty and environmental noise impact. USV in the class under-actuators when the number of control signals is less than the number of degrees of freedom of the vehicle's model. For the class system of under-actuator, the Hierarchy Sliding Mode Control (HSMC) method proves its effectiveness in documents [6], [7], [8].

Dynamic Positioning or Station Keeping is an essential control system for surface ships and for many other marine applications such as marine oil exploration, piping, automatic launch and recovery of an autonomous underwater vehicle [2]. In general, a dynamic positioning system refers to the control system of a surface ship, usually at low speed, in fullactuator mode, automatically maintaining its position and angle at a fixed point or preset working points.

* Corresponding author: ltranthang@gmail.com 
In this paper, proposed a controller uses (HSMC) method to control USV with underactuators model in the requirement for stable vehicle control at a given work position as consider environmental noise such as waves, winds and flows. The controller quality was performed in Matlab Simulink.

\section{Dynamic model of unmanned surface vehicle (USV)}

\subsection{Equations of Motion}

According to [1] the three degree of freedom model (3 DOF) (surge, sway and yaw) as:

$$
\left\{\begin{array}{c}
\dot{\dot{\eta}}=J(\underline{\eta}) \underline{v} \\
M \underline{\dot{v}}+C(\underline{v}) \underline{v}+D(\underline{v}) \underline{v}=\tau
\end{array}\right.
$$

where $\underline{\eta}=\left[\begin{array}{lll}x & y & \psi\end{array}\right]^{T}$ is the earth-fixed position and orientation vector; $\underline{v}=\left[\begin{array}{lll}u & v & r\end{array}\right]^{T}$ is the body-fixed translational and angular velocity vector;

$J(\underline{\eta})=\left[\begin{array}{ccc}\cos \psi & -\sin \psi & 0 \\ \sin \psi & \cos \psi & 0 \\ 0 & 0 & 1\end{array}\right] \quad$ is the coordinate

transformation between the body-fixed and earth-fixed coordinates; $M$ is a matrix sum of a rigid body mass matrix and an added mass matrix; $C(\underline{v})$ is a Coriolis matrix which includes the sum of a rigid body matrix

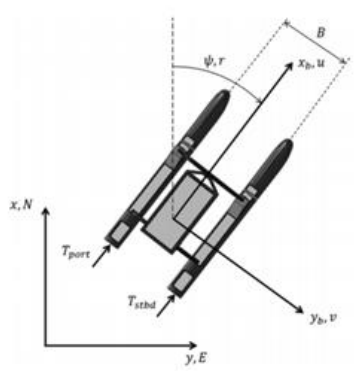

Fig. 1. SUV model and an added mass matrix; $D(\underline{v})$ is the addition of linear and nonlinear drag matrix; $\tau=\tau_{\text {wind }}+\tau_{\text {wave }}+\tau_{\text {all }}$ is the sum of the force and moment vectors acting on the USV; $\tau_{\text {wind }}, \tau_{\text {wave }}$ is part of the disturbance from the environment caused by wind, waves. This interference will not be known in advance $\tau_{n}(\underline{\eta}, \underline{v})$; $\tau_{\text {all }}=\left[\begin{array}{lll}\tau_{x} & 0 & \tau_{z}\end{array}\right]^{T}=\left[\begin{array}{lll}\left(\tau_{\text {port }}+\tau_{\text {stbd }}\right) & 0 & 0.5 B\left(\tau_{\text {port }}-\tau_{\text {stbd }}\right)\end{array}\right]^{T}$ is Vector of forces and moments created by the port and starboard side thrusters, $B$ is Beam overall.

Assume that the velocity of the water flow is $\underline{v}_{c}$ then $\underline{v}_{r}=\underline{v}_{-} \underline{v}_{c}=\left[\begin{array}{lll}u_{r} & v_{r} & r\end{array}\right]^{T}$ is relative velocity between the flow and movement of the USV. USV model is written as

$$
\left\{\begin{array}{c}
\dot{\dot{\eta}}=J(\underline{\eta}) \underline{v} \\
M \underline{\dot{v}}+C_{R B}(\underline{v}) \underline{v}+N\left(\underline{v}_{r}\right)=\tau_{a l l}
\end{array}\right.
$$

where $N\left(\underline{v}_{r}\right)=C_{A}\left(\underline{v}_{r}\right) \underline{v}_{r}+D\left(\underline{v}_{r}\right) \underline{v}_{r}$; 


$$
\begin{gathered}
M=\left[\begin{array}{ccc}
m-X_{\dot{u}} & 0 & -m y_{G} \\
0 & m-Y_{\dot{v}} & m x_{G}-Y_{\dot{r}} \\
-m y_{G} & m x_{G}-Y & I_{z}-N_{\dot{r}}
\end{array}\right] ; C_{A}\left(v_{r}\right)=\left[\begin{array}{ccc}
0 & 0 & Y_{\dot{v}} v_{r}+Y_{\dot{r}} r \\
0 & 0 & -X_{\dot{u}} u_{r} \\
-Y_{\dot{v}} v_{r}-Y_{\dot{r}} r & X_{\dot{u}} u_{r} & 0
\end{array}\right] ; \\
C_{R B}=\left[\begin{array}{ccc}
0 & 0 & -m\left(x_{G} r+v\right) \\
0 & 0 & -m\left(y_{G} r-u\right) \\
m\left(x_{G} r+v\right) & m\left(y_{G} r-u\right) & 0
\end{array}\right] ; \\
D_{A}\left(v_{r}\right)=-\left[\begin{array}{ccc}
X_{u}+X_{u|u|}\left|u_{r}\right| & 0 & 0 \\
0 & Y_{v}+Y_{v|v|}\left|v_{r}\right|+Y_{v|r|}|r| & Y_{r}+Y_{r|v|}\left|v_{r}\right|+Y_{r|r|}|r| \\
0 & N_{v}+N_{v|v|}\left|v_{r}\right|+N_{v|r|}|r| & N_{r}+N_{r|v|}\left|v_{r}\right|+N_{r|r|}|r|
\end{array}\right]
\end{gathered}
$$

$x_{G}, y_{G}$ is the center of gravity; $m$ is mass; $I_{z}$ Moment of inertia; $X_{u}, Y_{v}, N_{r}, X_{\dot{u}}, Y_{\dot{v}}$, $N_{\dot{v}}, Y_{\dot{r}}, N_{\dot{r}}$ are first order damping factors; $X_{u|u|}, Y_{v|v|}, N_{v|v|}, N_{r|r|}, Y_{r|r|}, Y_{v|r|}, N_{v|r|}, Y_{r|v|}$, $N_{r|v|}$ are the second-order modulus terms damping factors.

\subsection{Separation dynamics model}

Based on the transformation of Spong [9] for the model of an under-actuated system (2) in order to separate the system into two subsystems: a fully-actuated system and a free one. First of all to facilitate the transformation, we swap rows two and three of each vector $\tau_{\text {all }}, \underline{\eta}, \underline{v}$

$$
\tau_{\text {all }}=\left[\begin{array}{lll}
\tau_{x} & \tau_{z} & 0
\end{array}\right]^{T}, \underline{\eta}=\left[\begin{array}{lll}
x & \psi & y
\end{array}\right]^{T}, \underline{v}=\left[\begin{array}{lll}
u & r & v
\end{array}\right]^{T}
$$

Similar swapping rows two and three of the component matrices $J(\eta), M_{R B}, C_{R B}$, $C_{A}\left(v_{r}\right), D_{A}\left(v_{r}\right)$. Rewrite the position, velocity vectors, and the force, torque vectors of the system as:

$\underline{\eta}=\left[\begin{array}{ll}\underline{\eta}_{1} & \underline{\eta}_{2}\end{array}\right]^{T}$ with $\quad \underline{\eta}_{1}=\left[\begin{array}{ll}x & \Psi\end{array}\right]^{T}, \quad \underline{\eta}_{2}=y ; \quad \underline{v}=\left[\begin{array}{ll}\underline{v}_{1} & \underline{v}_{2}\end{array}\right]^{T}$ and $\quad \underline{v}_{1}=\left[\begin{array}{ll}u & r\end{array}\right]^{T}$,

$\underline{v}_{2}=v$

$\underline{v}_{r}=\left[\begin{array}{ll}\underline{v}_{r 1} & \underline{v}_{r 2}\end{array}\right]^{T}$ with $\underline{v}_{r 1}=\left[\begin{array}{ll}u_{r} & r\end{array}\right]^{T}, \underline{v}_{r 1}=v_{r} ; \tau_{\text {all }}=\left[\begin{array}{ll}\underline{\tau} & 0\end{array}\right]^{T}$ and $\underline{\tau}=\left[\begin{array}{ll}\tau_{x} & \tau_{z}\end{array}\right]^{T}$.

We obtain

$J=\left[\begin{array}{ll}J_{11} & J_{12} \\ J_{21} & J_{22}\end{array}\right]$ with $J_{11}=\left[\begin{array}{cc}\cos \psi & -\sin \psi \\ 0 & 0\end{array}\right], \quad J_{12}=\left[\begin{array}{l}0 \\ 1\end{array}\right], \quad J_{21}=\left[\begin{array}{ll}\sin \psi & \cos \psi\end{array}\right]$, $J_{22}=0$; 


$$
\begin{aligned}
& M=\left[\begin{array}{ll}
M_{11} & M_{12} \\
M_{21} & M_{22}
\end{array}\right] \quad \text { with } \quad M_{11}=\left[\begin{array}{cc}
m-X_{\dot{u}} & 0 \\
-m y_{G} & m x_{G}-N_{\dot{v}}
\end{array}\right], \quad M_{12}=\left[\begin{array}{c}
-m y_{G} \\
I_{z}-N_{\dot{r}}
\end{array}\right], \\
& M_{21}=\left[\begin{array}{ll}
0 & m-Y_{\dot{v}}
\end{array}\right], M_{22}=m x_{G}-Y_{\dot{r}} \text {; } \\
& C_{R B}=\left[\begin{array}{ll}
C_{R B 11} & C_{R B 12} \\
C_{R B 21} & C_{R B 22}
\end{array}\right] \quad \text { with } \quad C_{R B 11}=\left[\begin{array}{cc}
0 & 0 \\
m\left(x_{G} r+v\right) & m\left(y_{G} r-u\right)
\end{array}\right] \\
& C_{R B 12}=\left[\begin{array}{c}
-m\left(x_{G} r+v\right) \\
0
\end{array}\right], C_{R B 21}=\left[\begin{array}{ll}
0 & 0
\end{array}\right], C_{R B 22}=-m\left(y_{G} r-u\right) \text {; } \\
& C_{A}=\left[\begin{array}{ll}
C_{A 11} & C_{A 12} \\
C_{A 21} & C_{A 22}
\end{array}\right] \quad \text { with } \quad C_{A 11}=\left[\begin{array}{cc}
0 & 0 \\
-Y_{\dot{v}} v_{r}-Y_{\dot{r}} r & X_{\dot{u}} u_{r}
\end{array}\right], \quad C_{A 12}=\left[\begin{array}{c}
Y_{\dot{v}} v_{r}+Y_{\dot{r}} r \\
0
\end{array}\right] \text {, } \\
& C_{A 21}=\left[\begin{array}{ll}
0 & 0
\end{array}\right], C_{A 22}=-X_{\dot{u}} u_{r} \text {; } \\
& D_{A}=\left[\begin{array}{ll}
D_{A 11} & D_{A 12} \\
D_{A 21} & D_{A 22}
\end{array}\right] \text { with } D_{A 11}=\left[\begin{array}{cc}
X_{u}+X_{u|u|}\left|u_{r}\right| & 0 \\
0 & N_{v}+N_{v|v|}\left|v_{r}\right|+N_{v|r|}|r|
\end{array}\right] \text {, } \\
& D_{A 12}=\left[\begin{array}{c}
0 \\
N_{r}+N_{r|v|}\left|v_{r}\right|+N_{r|r|}|r|
\end{array}\right], \quad D_{A 21}=\left[\begin{array}{ll}
0 & Y_{v}+Y_{v|v|}\left|v_{r}\right|+Y_{v|r|}|r|
\end{array}\right] \text {, } \\
& D_{A 22}=Y_{r}+Y_{r|| \mid}\left|v_{r}\right|+Y_{r|r|}|r| \\
& N=\left[\begin{array}{ll}
N_{1} & N_{2}
\end{array}\right]^{T} \text { with } \quad N_{1}=M_{11} \underline{\dot{v}}_{r 1}+M_{12} \underline{\dot{v}}_{r 2}+\left(C_{A 11}+D_{A 11}\right) \underline{v}_{r 1}+\left(C_{A 12}+D_{A 12}\right) \underline{v}_{r 2}, \\
& N_{2}=M_{21} \underline{\dot{\underline{v}}}_{r 1}+M_{22} \underline{\dot{v}}_{r 2}+\left(C_{A 21}+D_{A 21}\right) \underline{v}_{r 1}+\left(C_{A 22}+D_{A 22}\right) \underline{v}_{r 2} \text {. }
\end{aligned}
$$

The dynamic model equation was rewritten as

$$
\left\{\begin{array}{c}
\underline{\dot{\eta}}_{1}=J_{11} \underline{\underline{v}}_{1}+J_{12} \underline{\underline{v}}_{2} \\
\underline{\dot{\eta}}_{2}=J_{21} \underline{\underline{v}}_{1}+J_{22} \underline{\underline{v}}_{2} \\
M_{11} \underline{\dot{v}}_{1}+C_{R B 1} \underline{\underline{v}}_{1}+M_{12} \underline{\underline{v}}_{2}+C_{R B 12} \underline{\underline{v}}_{2}+N_{1}=\underline{\tau} \\
M_{21} \underline{\dot{v}}_{1}+C_{R B 21} \underline{\underline{v}}_{1}+M_{22} \underline{\dot{v}}_{2}+C_{R B 22} \underline{\underline{v}}_{2}+N_{2}=0
\end{array}\right.
$$

From the 4th equation in the system (3)

$$
\underline{\dot{v}}_{2}=-M_{22}{ }^{-1}\left(M_{21} \underline{\dot{v}}_{1}+C_{R B 21} \underline{v}_{1}+C_{R B 22} \underline{v}_{2}+N_{2}\right) \text {. }
$$

Replace (4) in to the third equation in (3)

$$
M_{R B 11} \underline{\dot{v}}_{1}+C_{R B 11} \underline{v}_{1}-M_{R B 12} M_{22}^{-1}\left(M_{21} \underline{\dot{v}}_{1}+C_{21} \underline{v}_{1}+C_{22} \underline{v}_{2}+N_{2}\right)+C_{12} \underline{v}_{2}+N_{1}=\underline{\tau} .
$$

Equation (5) becomes

$$
\bar{M} \underline{\dot{v}}_{1}+\bar{C}_{1} \underline{v}_{1}+\bar{C}_{2} \underline{v}_{2}+\bar{N}=\underline{\tau} .
$$


Where $\bar{M}=M_{11}-M_{12} M_{22}{ }^{-1} M_{21} ; \bar{C}_{1}=C_{R B 11}-M_{12} M_{22}{ }^{-1} C_{R B 22} ; \bar{N}=N_{1}-M_{12} M_{22}{ }^{-1} N_{2}$

From (6) we have

$$
\underline{\dot{v}}_{1}=\bar{M}^{-1}\left(-\bar{C}_{1} \underline{v}_{1}-\bar{C}_{2} \underline{v}_{2}-\bar{N}\right)+\bar{M}^{-1} \underline{\tau} .
$$

Replace (7) in to (4)

$$
\underline{\dot{v}}_{2}=-M_{22}{ }^{-1}\left[M_{21} \bar{M}^{-1}\left(-\bar{C}_{1} v_{1}-\bar{C}_{2} v_{2}-\bar{N}\right)+C_{R B 21} \underline{\underline{v}}_{1}+C_{R B 22} \underline{v}_{2}+N_{2}\right]-M_{22}{ }^{-1} M_{21} \bar{M}^{-1} \underline{\tau} .
$$

Substituting (4) and (8) into (3), we have USV model as

$$
\left\{\begin{array}{c}
\dot{\underline{\eta}}_{1}=J_{11} \underline{\underline{v}}_{1}+J_{12} \underline{\underline{v}}_{2} \\
\underline{\dot{v}}_{1}=\bar{M}^{-1}\left(-\bar{C}_{1} \underline{\underline{v}}_{1}-\bar{C}_{2} \underline{\underline{v}}_{2}-\bar{N}\right)+\bar{M}^{-1} \underline{\tau} \\
\underline{\dot{\eta}}_{2}=J_{21} \underline{\underline{v}}_{1}+J_{22} \underline{v}_{2} \\
\underline{\dot{v}}_{2}=-M_{22}{ }^{-1}\left[M_{21} \bar{M}^{-1}\left(-\bar{C}_{1} v_{1}-\bar{C}_{2} v_{2}-\bar{N}\right)+C_{R B 21} \underline{v}_{1}+C_{R B 22} \underline{v}_{2}+N_{2}\right]-M_{22}{ }^{-1} M_{21} \bar{M}^{-1} \underline{\tau}
\end{array}\right.
$$

Model (9) it is perfectly suited to use the Hierarchy Sliding Mode Control (HSMC) controller to design the controller.

\section{Design the station keeping controller for USV}

The goal of the problem is control the position and heading angle of the USV keeping at a desire working point through the position and velocity vectors $\underline{\eta}$ and $\underline{v}$.

Rewrite (9) as general form

$$
\left\{\begin{array}{c}
\dot{\dot{\eta}}_{1}=J_{11} \underline{v}_{1}+J_{12} \underline{v}_{2} \\
\underline{\dot{v}}_{1}=f_{1}(X)+g_{1}(X) \underline{\tau} \\
\dot{\underline{\eta}}_{2}=J_{21} \underline{v}_{1}+J_{22} \underline{v}_{2} \\
\underline{\dot{v}}_{2}=f_{2}(X)+g_{2}(X) \underline{\tau}
\end{array}\right.
$$

where

$$
\begin{gathered}
X=\left[\begin{array}{llll}
\underline{\eta}_{1} & \underline{v}_{1} & \underline{\eta}_{2} & \underline{v}_{2}
\end{array}\right]^{T} ; g_{1}(X)=-M_{22}{ }^{-1} M_{21} \bar{M}^{-1} ; \\
f_{1}(X)=\bar{M}^{-1}\left(-\bar{C}_{1} \underline{v}_{1}-\bar{C}_{2} \underline{v}_{2}-\bar{N}\right) ; g_{1}(X)=\bar{M}^{-1} ; \\
f_{2}(X)=-M_{22}{ }^{-1}\left[M_{21} \bar{M}^{-1}\left(-\bar{C}_{1} v_{1}-\bar{C}_{2} v_{2}-\bar{N}\right)+C_{R B 21} \underline{v}_{1}+C_{R B 22} \underline{v}_{2}+N_{2}\right] .
\end{gathered}
$$

Definition of the error vector between the output and references signal is as follows

$$
e(t)=\left[\begin{array}{llll}
\underline{e}_{1} & \underline{e}_{2} & \underline{e}_{3} & \underline{e}_{4}
\end{array}\right]^{T}=\left[\begin{array}{llll}
\left(\underline{\eta}_{1}-\underline{\eta}_{1 d}\right) & \underline{v}_{1} & \left(\underline{\eta}_{2}-\underline{\eta}_{2 d}\right) & \underline{v}_{2}
\end{array}\right]^{T}
$$

Define sliding surfaces

$$
\left\{\begin{array}{l}
s_{1}=k_{1} \underline{e}_{1}+\underline{e}_{2} \\
s_{2}=k_{2} \underline{e}_{3}+\underline{e}_{4} \\
\underline{S}=\lambda s_{1}+\beta s_{2}
\end{array}\right.
$$

where

$k_{1}=\operatorname{diag}\left(k_{11} \quad k_{12}\right) \in R^{2 \times 2} ; k_{2} \in R, \lambda=\operatorname{diag}\left(\lambda_{1} \quad \lambda_{2}\right) \in R^{2 \times 2} ; \beta=\left[\begin{array}{ll}\beta_{1} & \beta_{2}\end{array}\right]^{T} \in R^{2 \times 1}$ and $k_{11}, k_{12}, k_{2}, \lambda_{1}, \lambda_{2}, \beta_{1}, \beta_{2}>0$. 
According to the Hierarchy Sliding Mode Control (HSMC) method for a system underactuator, the signal of the controller is divided into two components:

$$
\tau=\tau_{e q}+\tau_{s \mathrm{w}}
$$

with $\tau_{e q}$ is the signal used to control the subsystem; $\tau_{s \mathrm{w}}$ The signal used to control of the system's sliding surface.

Proof of system stability with HSMC controller is shown below.

Take the Lyapunov function for the closed system

$$
\begin{aligned}
& V=\frac{1}{2} \underline{S}^{T} \underline{S} \\
& \dot{V}=\underline{S}^{T} \cdot \underline{\dot{S}}
\end{aligned}
$$

From (11), (12) and (15) we obtain

$$
\begin{aligned}
& \dot{V}=\underline{S}^{T}\left[\lambda \dot{s}_{1}+\beta \dot{s}_{2}\right] \\
& =\underline{S}^{T}\left[\lambda\left(k_{1} J_{11} \underline{v}_{1}+k_{1} J_{12} \underline{v}_{2}+f_{1}+g_{1} \tau_{2}-k_{1} \dot{x}_{1 d}\right)+\right. \\
& \left.\quad \beta\left(k_{2} J_{21} \underline{v}_{1}+k_{2} J_{22} \underline{v}_{2}+f_{2}+g_{2} \tau_{2}-k_{2} \dot{x}_{3 d}\right)\right]
\end{aligned}
$$

$x_{1 d}, x_{3 d}$ are constants. Therefore $\dot{x}_{1 d}=\dot{x}_{3 d}=0$.

$$
\begin{aligned}
\dot{V} & =\underline{S}^{T} \cdot\left[\lambda\left(k_{1} J_{11} \underline{v}_{1}+k_{1} J_{12} \underline{v}_{2}+f_{1}+g_{1} \tau_{2}\right)+\beta\left(k_{2} J_{21} \underline{v}_{1}+k_{2} J_{22} \underline{v}_{2}+f_{2}+g_{2} \tau_{2}\right)\right] \\
= & \underline{S}^{T} \cdot\left[\begin{array}{l}
\lambda\left(k_{1} J_{11} \underline{v}_{1}+k_{1} J_{12} \underline{v}_{2}+f_{1}+g_{1}\left(\tau_{e q 1}+\tau_{s \mathrm{w} 1}+\tau_{e q 2}+\tau_{s \mathrm{w} 2}\right)\right) \\
+\beta\left(k_{2} J_{21} \underline{v}_{1}+k_{2} J_{22} \underline{v}_{2}+f_{2}+g_{2}\left(\tau_{e q 1}+\tau_{s \mathrm{w} 1}+\tau_{e q 2}+\tau_{s \mathrm{w} 2}\right)\right)
\end{array}\right] \\
= & \underline{S}^{T} \cdot\left[\begin{array}{l}
\lambda\left(k_{1} J_{11} \underline{v}_{1}+k_{1} J_{12} \underline{v}_{2}+f_{1}+g_{1} \tau_{e q 1}\right)+\beta\left(k_{2} J_{21} \underline{v}_{1}+k_{2} J_{22} \underline{v}_{2}+f_{2}+g_{2} \tau_{e q 2}\right) \\
+\tau_{s \mathrm{w} 1}\left(\lambda g_{1}+\beta g_{2}\right)+\tau_{s \mathrm{w} 2}\left(\lambda g_{1}+\beta g_{2}\right) \\
+\lambda g_{1} \tau_{e q 2}+\beta g_{2} \tau_{e q 1}+k \cdot \underline{S}+\delta \operatorname{sign}(\underline{S})-(k \cdot \underline{S}+\delta \operatorname{sign}(\underline{S}))
\end{array}\right]
\end{aligned}
$$

where: $k=\operatorname{diag}\left(k_{x} k_{y}\right), k_{x}, k_{y}>0 ; \delta=\operatorname{diag}\left(\begin{array}{ll}\delta_{1} & \delta_{2}\end{array}\right), \delta_{1}, \delta_{2}>0$.

By applying the principle of stability Lyapunov, we choose such control signals $d V / d t$ define negative

$$
\left\{\begin{array}{c}
\tau_{e q 1}=\frac{-\left(k_{1} J_{11} \underline{v}_{1}+k_{1} J_{12} \underline{v}_{2}+f_{1}\right)}{g_{1}} \\
\tau_{e q 1}=\frac{-\left(k_{2} J_{21} \underline{v}_{1}+k_{2} J_{22} \underline{v}_{2}+f_{2}\right)}{g_{2}} \\
\tau_{s \mathrm{w} 2}=-\tau_{s \mathrm{w} 1}-\frac{\lambda g_{1} \tau_{e q 2}+\beta g_{2} \tau_{e q 1}}{\lambda g_{1}+\beta g_{2}}-\frac{k \cdot \underline{S}+\delta \operatorname{sign}(\underline{S})}{\lambda g_{1}+\beta g_{2}}
\end{array}\right.
$$

Substituting (18) into (17), we obtain $\frac{d V}{d t}=\underline{S}^{T} \cdot \underline{\dot{S}}=-\underline{S}^{T}(k \cdot \underline{S}+\delta \operatorname{sign}(\underline{S})) \leq 0$

The control signal is determined as 


$$
\begin{gathered}
\underline{\tau}=\tau_{e q 1}+\tau_{s \mathrm{w} 1}+\tau_{e q 2}+\tau_{s \mathrm{w} 2} \\
=-\frac{\lambda f_{1}+\beta f_{2}+\lambda\left(k_{1} J_{11} \underline{v}_{1}+k_{1} J_{12} \underline{v}_{2}\right)+\beta\left(k_{2} J_{21} \underline{v}_{1}+k_{2} J_{22} \underline{v}_{2}\right)+k \cdot \underline{S}+\delta \operatorname{sign}(\underline{S})}{\lambda g_{1}+\beta g_{2}}
\end{gathered}
$$

Remark. In the control signal formula above we note that this design method needs to know the model. On the other hand, there are many constants that need to be chosen suitable value for each specific model. Therefore, we will have to have simulation steps to select the appropriate constants for the controller. These problems will be minimized when we design a controller that can adapt to unstable in system parameters.

\section{Simulation Results}

The simulation results were performed on Matlab Simulink. With the ship parameters of USV shown in [1]. Desired position in the x-axis is $3(\mathrm{~m})$, in the y-axis is $2(\mathrm{~m})$, the value of the heading angle is $0.2(\mathrm{rad})$, Considering the effect of flow as a function of time as follows $\underline{v}_{c}=\left[\begin{array}{lll}0.8 \sin (0.7 t) & 0.2 \sin (0.5 t) & 0\end{array}\right]^{T}(\mathrm{~m} / \mathrm{s})$.

(a)

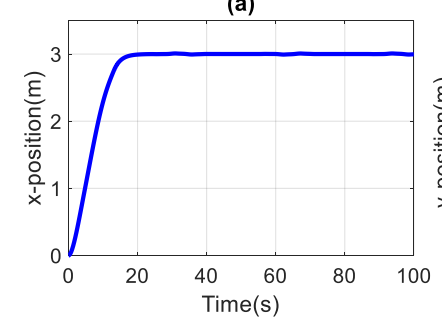

(b)

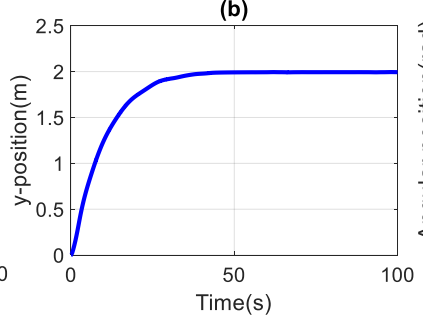

(c)

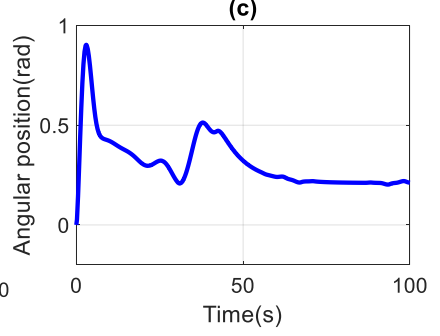

Fig. 2. USV's position in the case effect of flow without wind or wave interference

(a)

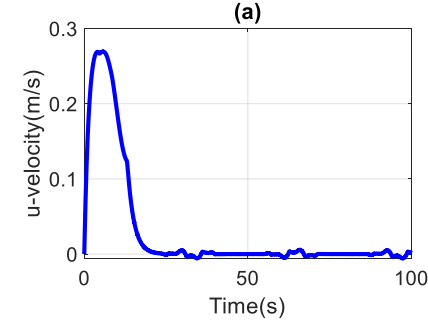

(b)

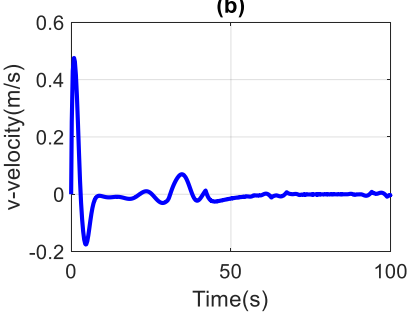

(c)

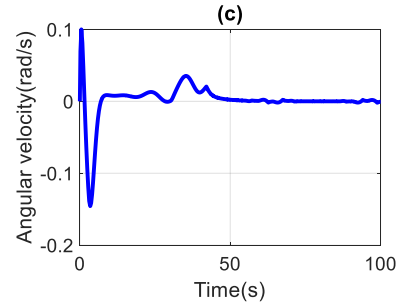

Fig. 3. USV's velocity in case of only considering flow effect

In the case of considering interference, wind has the form referenced in [10] as follows $\tau_{n}(\underline{\eta}, \underline{v})=\left[\begin{array}{lll}2|u|^{2}+0.05 & |r|^{3}+\sin (v) & 0\end{array}\right]^{T}$.

Desired position setting in the $\mathrm{x}$-axis is $4(\mathrm{~m})$, in the y-axis $2(\mathrm{~m})$, the value for the heading angle is $-0.15(\mathrm{rad})$. 

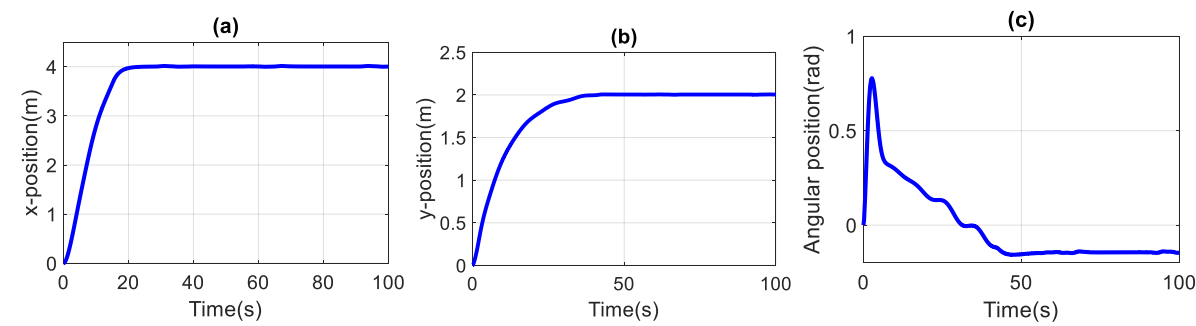

Fig. 4. SUV's position in case of flow impact and effect of wind and waves

(a)

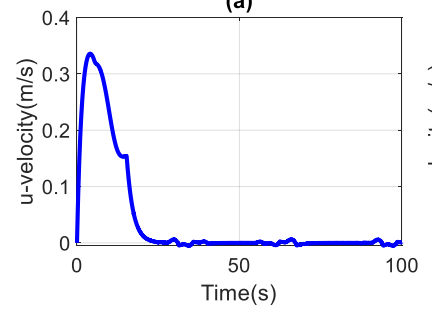

(b)

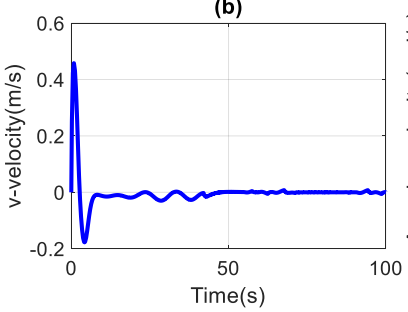

(c)

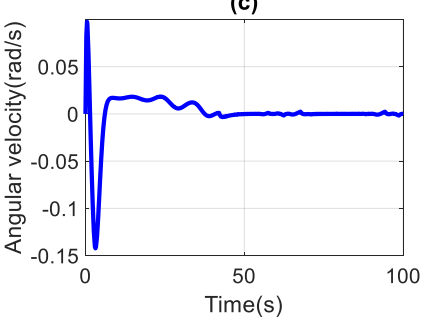

Fig. 5. SUV's speed in case of flow impact and effect of wind and waves

The simulation results from figure 2, 3, 4, 5 show that the quality of the Hierarchy Sliding Mode Control controller for USV is very good with the time specified in the X-direction is 20s, in the $y$-direction is $40 \mathrm{~s}$ and in the angle is $43 \mathrm{~s}$, the over-adjustment and the setting error are small. The system responds well to the set value and stability with effects of flows (Figure 2,3) and when considering interference from waves and winds (Figure 4, 5).

\section{Conclusions}

The paper has proposed a controller for USV with an under-actuator model using the model transformation to suitable form to apply controller design (HSMC) for USV with the excellent deal with dynamic stability in conditions where the environmental disturbances affect. The simulation results show that the proposed controller with the transient time and error established as well as the overshoot of small value. As a result, the controller designed in this article helps the system (USV) operate stably and reduce energy loss even when subjected to external influences.

\section{References}

1. Alejandro G G, Ivana C G, David B A and Leonardo G 2020 Proc. Int. Conf of the International Conference on Deep Learning Technologies. $4^{\text {th }}$ (ICDLT 2020) p 118122.

2. Wilhelm B. Klinger, Ivan R. Bertaska, Karl D. von Ellenrieder and M. R. Dhanak Journal of Oceanic Engineering. 42. (2016)

3. Zaopeng D, Lei W, Yueming L, Tao L and Guocheng Z International Journal of Naval Architecture and Ocean Engineering 7, 817. (2015) 
4. Tao L, Zaopeng D, Hongwang D, Lifei S and Yunsheng M. Polish Maritime Research, 24, 3. (2017)

5. Arvind P, Jnaneshwar D, Gaurav S S 2008 Int. Conf on Intelligent Robots and Systems (Nice, France).

6. Yao Z 2016. Int. J. Robust Nonlinear Control Nonlinear robust adaptive hierarchical sliding mode control approach for quadrotors (Wiley Online Library).

7. Long Ch, Hai W, Yunzhi H, Zhaowu P, Ming Y, Xuefeng Z, Mao Y and Youhao H Int. J. Mechanical Systems and Signal Processing, 139 (2020)

8. Wei L, Si-yi Ch, and Hui-xian H Int. J of Control, Automation and Systems 18, 339, (2019)

9. Spong M W Engineering, Computer Science; Proc. Int. Conf on Intelligent Robots and Systems, 314 (1994)

10. Shi L D, Cong W and Fei L IEEE Transactions on Industrial Informatics, 8 (4), 801 (2012)

11. Huang H J. Ocean Engineering. 133, 215. (2017) 